\title{
The Finite Difference Methods for Hyperbolic - Parabolic Equations
}

\author{
Abbas Y. Al-Bayati Saad A. Manaa Ekhlass S. Al-Rawi \\ profabbasalbayati@yahoo.com_drekhlass-alrawi@uomosul.edu.iq \\ College of Computer Sciences and Mathematics \\ University of Mosul/Iraq
}

Received on: 12/04/2005

Accepted on: 30/05/2005

\section{ABSTRACT}

The objective of this paper is to construct numerical schemes using finite difference methods for the one-dimensional general hyperbolicparabolic- reaction problem.

The finite difference method with the exponential transformation form is used to solve the problem, and employs difference approximation technique to obtain the numerical solutions. Computational examples are presented and compared with the exact solutions. We obtained that the Crank-Nicholson scheme is more accurate than Forward scheme. Therefore the form of exponential transformation for the problem yields a stable solution compared with exact solution.

Keywords: Finite difference methods; Exponential transformation; CrankNicholson scheme; Hyperbolic- parabolic- reaction problem.

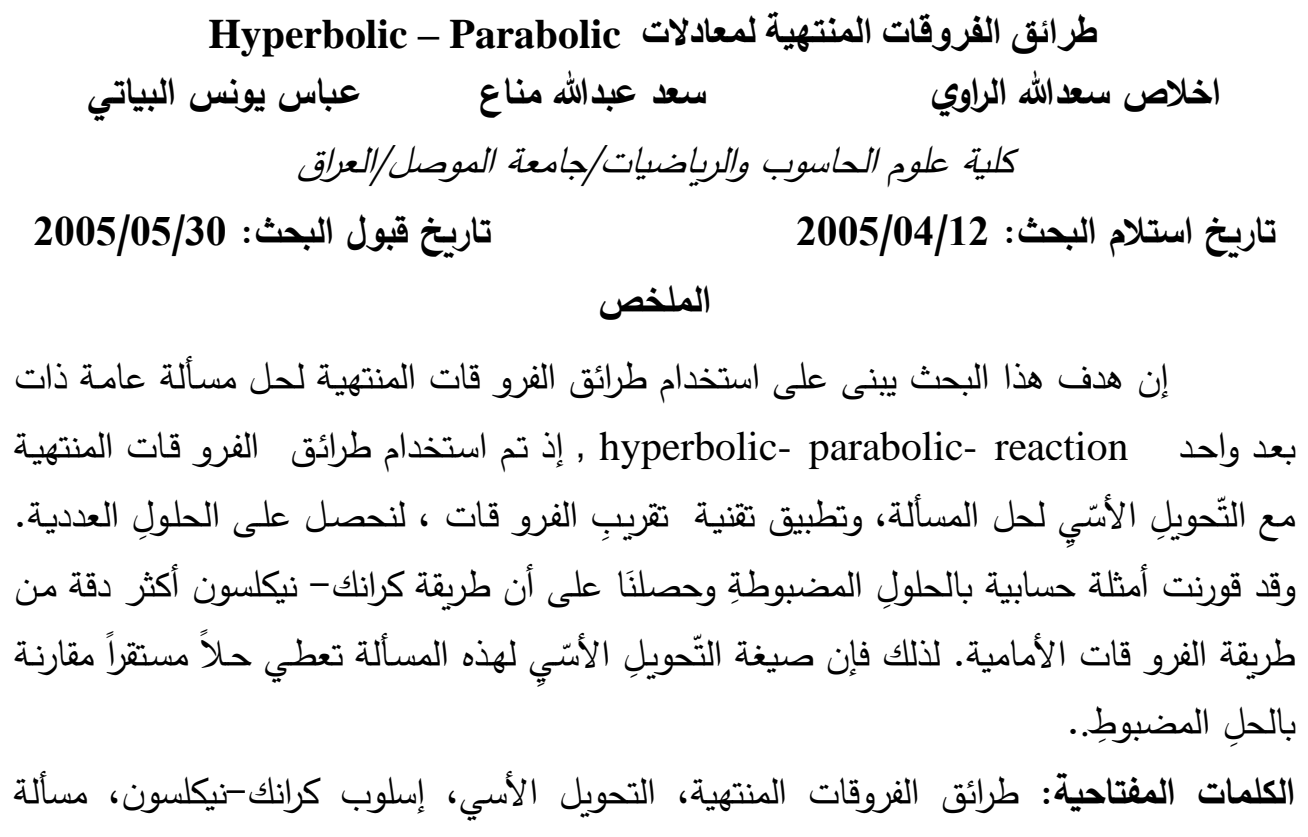

. hyperbolic- parabolic- reaction 


\section{Introduction:}

Various finite difference algorithms or schemes have been presented for the solution of hyperbolic- parabolic problem or its simpler derivatives, such as the classical diffusion equation .It is well-known that many of these schemes are partially unsatisfactory due to the formation of oscillations and numerical diffusion within the solutions (Smith et al.,1973). Solution by the finite difference method, although more general, will involve stability and convergence problems, may require special handling of boundary conditions, and may require large computer storage and execution time. The problem of numerical dispersion for finite difference solutions is also difficult to overcome (Guymon,1970).

The hyperbolic-parabolic equation is a linear, nonsymmetrical partial differential equation of the parabolic type. The nonsymmetry arises from the so-called hyperbolic terms $\frac{\partial(v \psi)}{\partial x}$, which have been the principle source of difficulty in numerical solution of problems of this type. The mathematical literature relating to the solution of partial differential equations is concerned primarily with symmetrical problems and scant attention has been paid to nonsymmetrical equations, an increasingly important class of problems considering modern preoccupation with the quality of the environment (Riddaway, 2001 and Guymon et al.,1970).

Price et al.(1968) solved the one-dimensional diffusion-convection equation by several techniques including conventional finite differences, and the method of characteristics. The extension of this latter method to two dimensions was mentioned but no solutions were given.

Smith et al. (1973) used Rayleigh-Ritz finite elements for diffusionconvection problems and discussed the accuracy and stability of the techniques.

Chen et al. (1998) developed higher-order Godunov method for one hyperbolic- parabolic-reaction problems, derived an error estimation and presented computational examples. Garbey et al. (2001) presented a fast algorithm for numerical solution of systems of reaction-diffusion equations.

Bindu (2002) investigated the integrability properties of the generalized Fisher type equation to obtain physically interesting solution using Lie symmetry analysis.

In this paper, the authors, using the exponential transformation, treat the classical differential representation for one-dimensional hyperbolicparabolic- reaction problems, and employ difference approximation 
techniques to obtain the numerical solutions. The comparison with the exact solutions shows good numerical approximations in the two examples.

\section{The Mathematical Model:}

The one-dimensional transport equation of a general hyperbolicparabolic- reaction problem of the form (see Chen et al. ,1998)

$$
\frac{\partial \psi}{\partial t}+\frac{\partial}{\partial x}\left[-D \frac{\partial \psi}{\partial x}+v \psi\right]=S(\psi) \quad, \quad x \in[0, L], v>0, D \geq 0
$$

where $S(\psi)$ is a linear function of $\psi$, the boundary conditions are

$$
\begin{array}{ll}
\psi(0, t)=\psi_{0}(t) & , t>0 \\
\psi(L, t)=\psi_{L}(t) \text { or } \frac{\partial \psi(x, t)}{\partial x} I_{x=L}=0 & , t>0
\end{array}
$$

and the initial condition is

$$
\psi(x, 0)=f(x) \quad, \quad 0 \leq x \leq L
$$

Equation (1) with its auxiliary conditions is an approximate mathematical model of some physical problem. They make use of the fact that although (1) is nonsymmetrical, any second-order partial differential equation can be rendered symmetrical by the use of a transformation. In this case of (1), the transformation is simply (see Smith et al. ,1973)

$$
\psi=H \exp (v \chi / 2 D)
$$

which after taking first and second derivatives and substituting into equation (1) yields the following transformed partial differential equation:

$$
\begin{aligned}
& \frac{\partial H}{\partial t} \exp (v \chi / 2 D)-D\left[\frac{v^{2}}{4 D^{2}} H \exp (v \chi / 2 D)-\frac{v}{2 D} \frac{\partial H}{\partial x} \exp (v \chi / 2 D)+\frac{\partial^{2} H}{\partial x^{2}} \exp (v \chi / 2 D)\right. \\
& \left.+\frac{v}{2 D} \frac{\partial H}{\partial x} \exp (v \chi / 2 D)\right]+v\left[\frac{v}{2 D} H \exp (\nu \chi / 2 D)+\frac{\partial H}{\partial x} \exp (\nu \chi / 2 D)\right]=S(H \exp (v \chi / 2 D))
\end{aligned}
$$

Hence

$$
\frac{\partial H}{\partial t}-D \frac{\partial^{2} H}{\partial x^{2}}+\frac{v^{2}}{4 D} H=S(H) \quad, x \in[0, L], v>0, D>0
$$

The transformed geometric boundary condition becomes

$$
H(0, t)=\psi_{0}(t) \quad t>0
$$


The transformed natural boundary condition is

$$
H(L, t)=\exp (-v L / 2 D) \psi_{L}(t) \text { or }\left.\left(\frac{\partial H}{\partial x}+\frac{v H}{2 D}\right)\right|_{x=L}=0, t>0
$$

Finally, the initial condition transforms to

$$
H(x, 0)=f(x) \exp (-v x / 2 D) \quad, \quad 0 \leq x \leq L
$$

\section{Numerical Solution by the Finite Difference Methods:}

We consider the approximate solution of the problem and its transformation by using finite difference equations (FDE). That is we will use two methods: forward finite difference method (explicit method) and Crank-Nicholson method (implicit method) to solve these problems.

We introduce a uniform grid by defining the following discrete set of points in the $\mathrm{x}, \mathrm{t}$ plane:

and

$$
\mathrm{x}_{\mathrm{i}}=\mathrm{ih}, \quad \mathrm{i}=0,1, \ldots, \mathrm{n}-1, \mathrm{n}
$$

$$
\mathrm{t}_{\mathrm{j}}=\mathrm{jk}, \quad \mathrm{j}=0,1, \ldots, \mathrm{m}-1, \mathrm{~m}
$$

The grid functions $\psi$ and $\mathrm{H}$ in our finite difference approximations are denoted by $\psi_{i, j}=\psi\left(x_{i}, t_{j}\right)$ and $H_{i, j}=H\left(x_{i}, t_{j}\right)$, respectively.

In order to solve equations (1) and (5) numerically, we replace the differential equations with an analogous difference equations. Firstly, we apply two finite difference methods for solving equation (1), and secondly, we also apply these methods for solving equation (5).

\subsection{Forward finite difference method (explicit method):}

We approximate the partial differential equation (1) by the finite difference quotients

$$
\frac{\psi_{i, j+1}-\psi_{i, j}}{k}-D \frac{\psi_{i-1, j}-2 \psi_{i, j}+\psi_{i+1, j}}{h^{2}}+v \frac{\psi_{i+1, j}-\psi_{i, j}}{h}=S\left(\psi_{i, j}\right)
$$

Where $i=0,1, \ldots, n-1, n$ and $j=0,1, \ldots, m-1, m$, then we have

$$
\psi_{i, j+1}=k S\left(\psi_{i, j}\right)+r \psi_{i-1, j}+\left(1+\frac{v k}{h}-2 r\right) \psi_{i, j}+\left(r-\frac{v k}{h}\right) \psi_{i+1, j}
$$

Also the difference equation of (5) is

$$
\frac{H_{i, j+1}-H_{i, j}}{k}-D \frac{H_{i-1, j}-2 H_{i, j}+H_{i+1, j}}{h^{2}}+\frac{v^{2}}{4 D} H_{i, j}=S\left(H_{i, j}\right)
$$


and then

$$
H_{i, j+1}=k S\left(H_{i, j}\right)+r H_{i-1, j}+\left(1-\frac{v^{2} k}{4 D}-2 r\right) H_{i, j}+r H_{i+1, j}
$$

Where $\mathrm{i}=0,1, \ldots, \mathrm{n}-1, \mathrm{n}$ and $\mathrm{j}=0,1, \ldots, \mathrm{m}-1, \mathrm{~m}$

And also $h$ is a step size of $x, k$ is a step size of $t$ and $r=\frac{k D}{h^{2}}$.

\subsection{Crank-Nicholson method (implicit method):}

We approximate the partial differential equation (1) by the finite difference quotients:

$\frac{\psi_{i, j+1}-\psi_{i, j}}{k}-D \frac{\psi_{i-1, j+1}-2 \psi_{i, j+1}+\psi_{i+1, j+1}+\psi_{i-1, j}-2 \psi_{i, j}+\psi_{i+1, j}}{2 h^{2}}+v \frac{\psi_{i+1, j}-\psi_{i, j}}{h}=S\left(\psi_{i, j}\right)$

Where $i=0,1, \ldots, n-1, n$ and $j=0,1, \ldots, m-1, m$, then we have

$$
\begin{aligned}
& -\psi_{i-1, j+1}+\left(\frac{2}{r}+2\right) \psi_{i, j+1}-\psi_{i+1, j+1}=\frac{2 h^{2}}{D} S\left(\psi_{i, j}\right) \\
& +\psi_{i-1, j}+\left(\frac{2 v h}{D}+\frac{2}{r}-2\right) \psi_{i, j}+\left(1-\frac{2 v h}{D}\right) \psi_{i+1, j}
\end{aligned}
$$

Also the difference equation of (5) is

$$
\frac{H_{i, j+1}-H_{i, j}}{k}-D \frac{H_{i-1, j+1}-2 H_{i, j+1}+H_{i+1, j+1}+H_{i-1, j}-2 H_{i, j}+H_{i+1, j}}{2 h^{2}}+\frac{v^{2}}{4 D} H_{i, j}=S\left(H_{i, j}\right)
$$

then

$$
\begin{aligned}
& -H_{i-1, j+1}+\left(\frac{2}{r}+2\right) H_{i, j+1}-H_{i+1, j+1}=\frac{2 h^{2}}{D} S\left(H_{i, j}\right) \\
& +H_{i-1, j}+\left(\frac{2}{r}-\frac{v^{2} h^{2}}{2 D^{2}}-2\right) H_{i, j}+H_{i+1, j}
\end{aligned}
$$

Where $i=0,1, \ldots, n-1, n$ and $j=0,1, \ldots, m-1, m$

And also $h$ is step size of $x, k$ is step size of $t$ and $r=\frac{k D}{h^{2}}$. 


\section{Computational Examples:}

In this section, we have solved two different problems and we have compared between them using the transformation in (4). We compare the results with the exact solutions by using MATLAB language.

\subsection{Hyperbolic- parabolic problem:}

The first test problem is chosen from Peaceman (1977). It is a onedimensional linear unsteady hyperbolic- parabolic problem described by

$\frac{\partial u}{\partial t}=D \frac{\partial^{2} u}{\partial x^{2}}-v \frac{\partial u}{\partial x} \quad, \quad v=1, D=0.06$

with the initial condition

$$
u(x, 0)=0 \quad, \quad x>0,
$$

and the boundary conditions

$$
u(0, t)=1 \quad \text { and } u(\infty, t)=0, \quad t>0
$$

The analytical solution of this problem is given by Karamouzis (1990)

$$
u(x, t)=\frac{1}{2} \operatorname{erfc}\left[\frac{x-v t}{2(D t)^{\frac{1}{2}}}\right]+\frac{1}{2} \exp (v x / D) \operatorname{erfc}\left[\frac{x+v t}{2(D t)^{\frac{1}{2}}}\right]
$$

where erfc is the complementary error function, defined by:

$\operatorname{erfc}(x)=1-\operatorname{erf}(x)$

erf $(\mathrm{x})$ is the error function and is defined as:

$\operatorname{erf}(x)=\frac{2}{\sqrt{\pi}} \int_{0}^{x} e^{-t^{2}} d t$

The value of the error function could be expressed in a series form, Abramowitz (1965).

$\operatorname{erf}(x)=\frac{2}{\sqrt{\pi}}\left(x-\frac{x^{3}}{3}+\frac{x^{5}}{2 !(5)}-\frac{x^{7}}{3 !(7)}+\ldots\right)$

All Figures $(1,2,3,4)$ illustrate the numerical solutions of schemes (3.1) and (3.2) without and with transform of the problem (4.1), respectively. A comparison between the numerical approximations and the exact solution at $t=0.45$ is given in figure (6) and table (1), which shows good numerical approximations by using exponential transformation for the problem. That is when we increase the value of time $t$ we get a smooth curve by using exponential transformation method which means that it is more accurate than without this transform. 


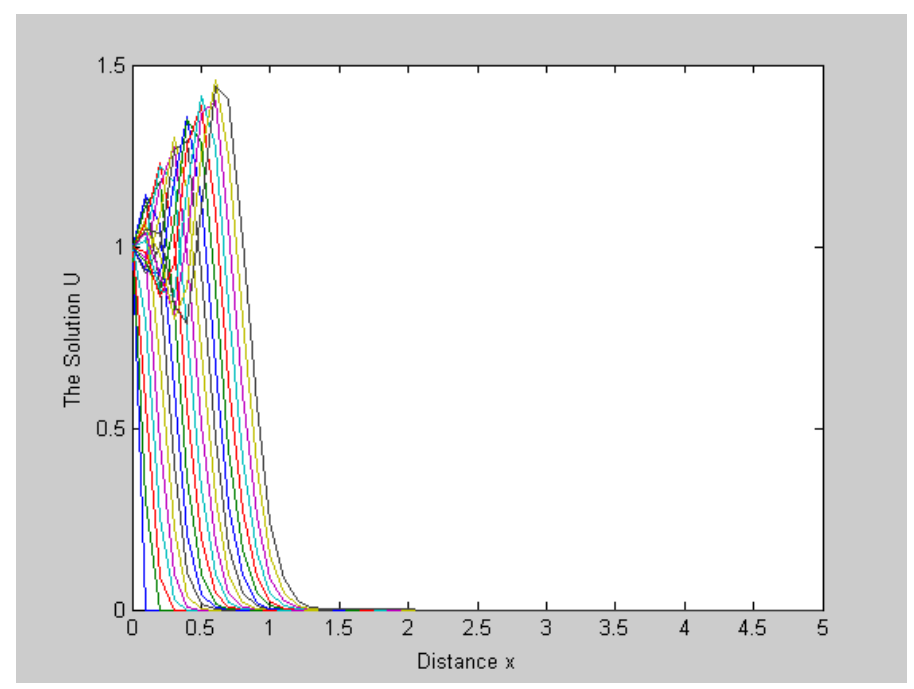

Fig.(1): Numerical approximation of the Forward F.D.M. for the first problem

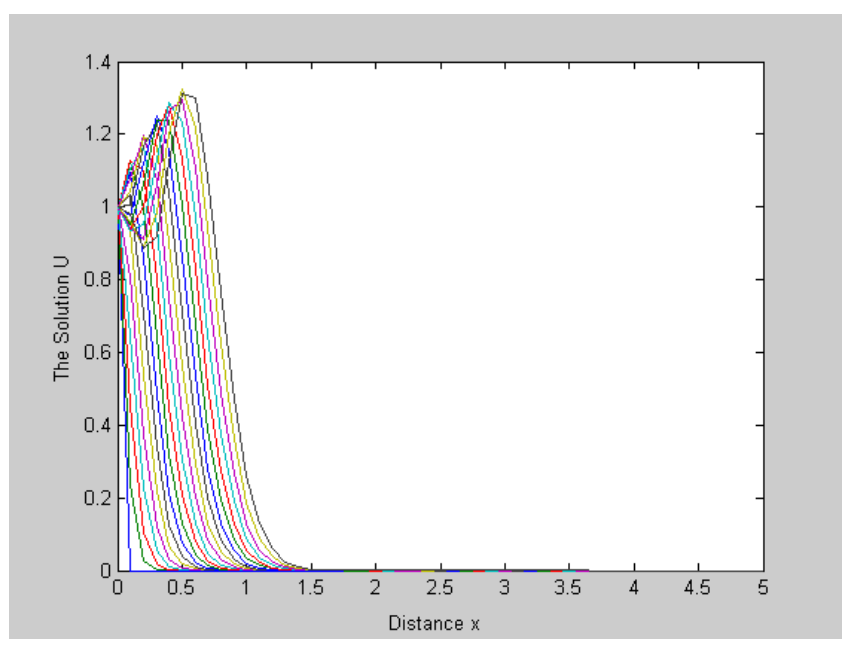

Fig.(2): Numerical approximation of the Crank-Nicolson F.D.M. for the first problem 


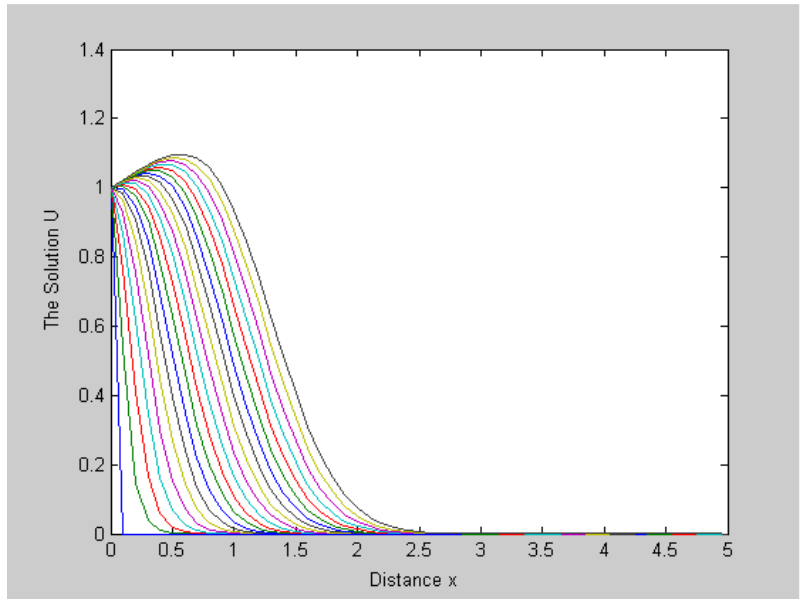

Fig.(3): Numerical approximation of the Forward F.D.M for the transformed first problem

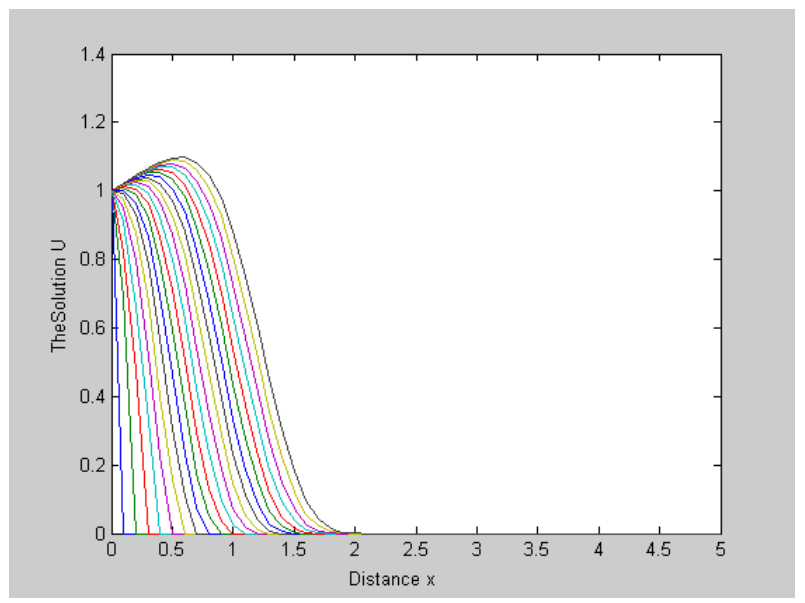

Fig.(4): Numerical approximation of the Crank-Nicolson F.D.M. for the transformed first problem 


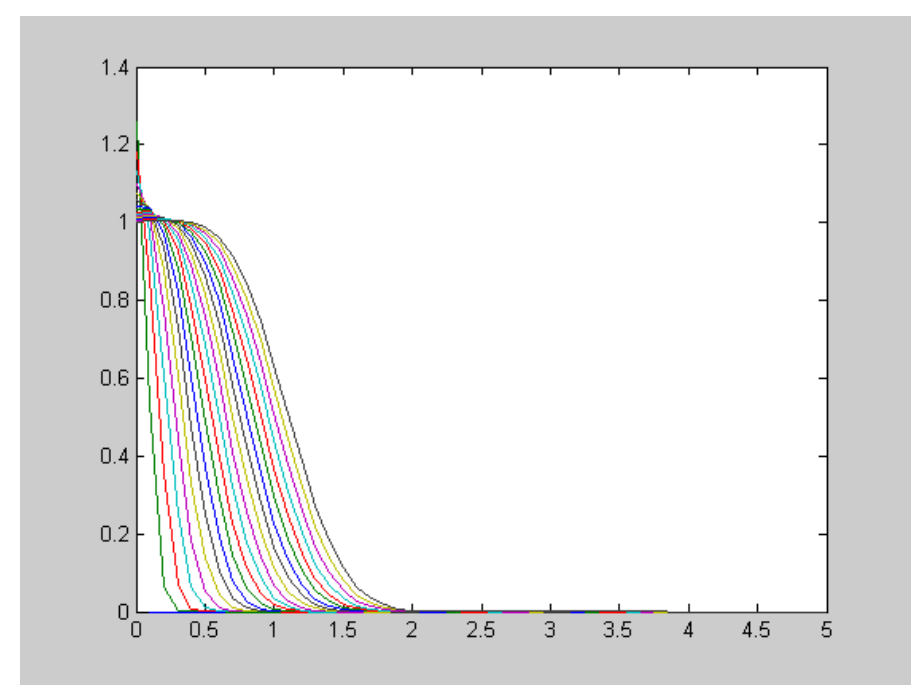

Fig.(5): The exact solution of the first problem

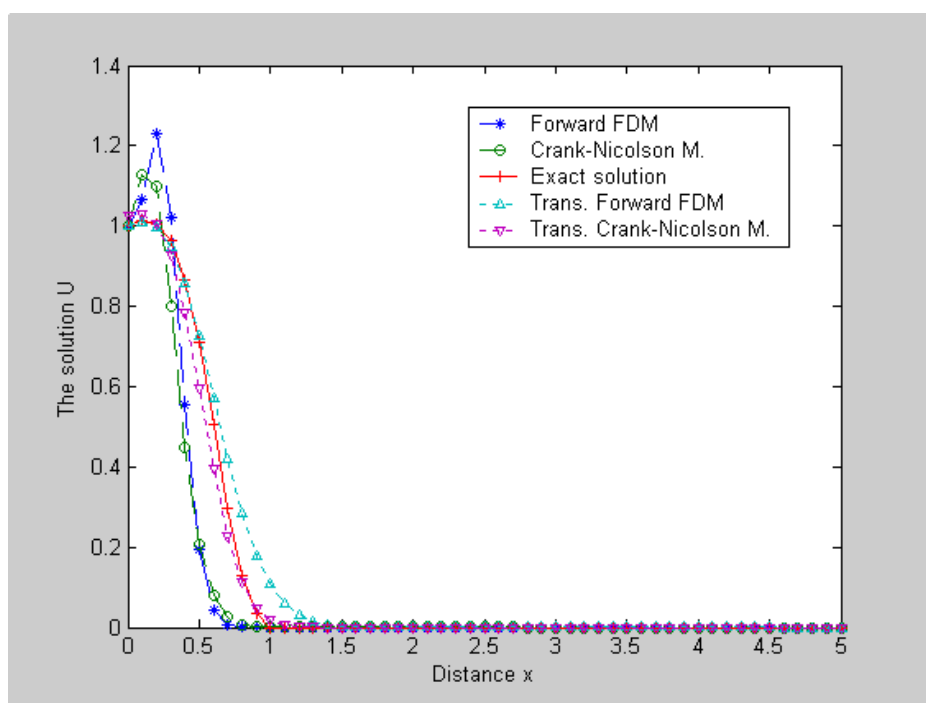

Fig.(6): Comparison between these F.D.M and the exact solution of the first problem at $\mathrm{t}=0.45$ 
Table (1): Numerical approximations of F.D.M. and exact solution of the first problem at $\mathrm{t}=0.45, \mathrm{~L}=5, \mathrm{~h}=0.1$, and $\mathrm{k}=0.05$

\begin{tabular}{|c|c|c|c|c|c|}
\hline $\mathrm{X}$ & $\begin{array}{l}\text { Forward } \\
\text { F.D.M. }\end{array}$ & $\begin{array}{l}\text { Crank-Nicolson } \\
\text { M. }\end{array}$ & $\begin{array}{l}\text { Trans. Forward } \\
\text { F.D.M. }\end{array}$ & $\begin{array}{l}\text { Trans. Crank- } \\
\text { Nicolson M. }\end{array}$ & $\begin{array}{l}\text { Exact } \\
\text { Solutio } \\
n\end{array}$ \\
\hline 0 & 1. $0000 \mathrm{e}+00$ & 1. $000000 \mathrm{e}+000$ & $1.0000000 \mathrm{e}+000$ & $1.0000000 \mathrm{e}+000$ & 1.0264 \\
\hline .1 & 1.067020 & 1.126276 & $1.0125566 \mathrm{e}+000$ & $1.0099174 \mathrm{e}+000$ & 1.0290 \\
\hline .2 & 1.228500 & 1.097644 & $1.0059737 \mathrm{e}+000$ & 9.9777153e-001 & 1.0035 \\
\hline .3 & 1.023324 & .8007645 & $9.63287341 \mathrm{e}-001$ & $9.4969569 \mathrm{e}-001$ & 0.9260 \\
\hline .4 & .5532953 & .4501546 & $8.66791942 \mathrm{e}-001$ & $8.5756869 \mathrm{e}-001$ & 0.7851 \\
\hline .5 & .1948131 & .2051848 & $7.09839004 \mathrm{e}-001$ & $7.2575634 \mathrm{e}-001$ & 0.5957 \\
\hline 6 & $.4467458 \mathrm{e}-1$ & $.7912419 \mathrm{e}-1$ & $5.06750795 \mathrm{e}-001$ & $5.7143682 \mathrm{e}-001$ & 0.3965 \\
\hline .7 & $.6464772 \mathrm{e}-2$ & $.2666623 \mathrm{e}-1$ & $2.98760643 \mathrm{e}-001$ & $4.1757679 \mathrm{e}-001$ & 0.2281 \\
\hline .8 & $.5380020 \mathrm{e}-3$ & $.8050579 \mathrm{e}-2$ & $1.30604734 \mathrm{e}-001$ & $2.8360939 \mathrm{e}-001$ & 0.1122 \\
\hline .9 & $.1968300 \mathrm{e}-4$ & $.2218941 \mathrm{e}-2$ & $3.55876988 \mathrm{e}-002$ & $1.7974974 \mathrm{e}-001$ & 0.0469 \\
\hline 1 & 0.0000 & $.5666747 \mathrm{e}-3$ & 0.0000 & $1.0688882 \mathrm{e}-001$ & 0.0166 \\
\hline 1.1 & 0.0000 & $.1356606 \mathrm{e}-3$ & 0.0000 & $5.9990546 \mathrm{e}-002$ & 0.0049 \\
\hline 1.2 & 0.0000 & $.3072918 \mathrm{e}-4$ & 0.0000 & $3.1963971 \mathrm{e}-002$ & 0.0012 \\
\hline 1.3 & 0.0000 & $.6635834 \mathrm{e}-5$ & 0.0000 & $1.6257023 \mathrm{e}-002$ & 0.0003 \\
\hline 1.4 & 0.0000 & $.1374550 \mathrm{e}-5$ & 0.0000 & $7.9318558 \mathrm{e}-003$ & 0.0000 \\
\hline 1.5 & 0.0000 & $.2745068 \mathrm{e}-6$ & 0.0000 & $3.7288364 \mathrm{e}-003$ & 0.0000 \\
\hline 1.6 & 0.0000 & $.5307784 \mathrm{e}-7$ & 0.0000 & $1.6955769 \mathrm{e}-003$ & 0.0000 \\
\hline 1.7 & 0.0000 & $.9972136 \mathrm{e}-8$ & 0.0000 & $7.4830411 \mathrm{e}-004$ & 0.0000 \\
\hline 1.8 & 0.0000 & $.1825954 \mathrm{e}-8$ & 0.0000 & $3.2146919 \mathrm{e}-004$ & 0.0000 \\
\hline 1.9 & 0.0000 & $.3266920 \mathrm{e}-9$ & 0.0000 & $1.3477929 \mathrm{e}-004$ & 0.0000 \\
\hline 2 & 0.0000 & $.5723982 \mathrm{e}-10$ & 0.0000 & $5.5272724 \mathrm{e}-005$ & 0.0000 \\
\hline 2.1 & 0.0000 & $.9840191 \mathrm{e}-11$ & 0.0000 & $2.2215882 \mathrm{e}-005$ & 0.0000 \\
\hline 2.2 & 0.0000 & $.1662575 \mathrm{e}-11$ & 0.0000 & $8.7667394 \mathrm{e}-006$ & 0.0000 \\
\hline 2.3 & 0.0000 & $.2764825 \mathrm{e}-12$ & 0.0000 & $3.4017521 \mathrm{e}-006$ & 0.0000 \\
\hline 2.4 & 0.0000 & $.4531306 \mathrm{e}-13$ & 0.0000 & $1.2997183 \mathrm{e}-006$ & 0.0000 \\
\hline 2.5 & 0.0000 & $.7327305 \mathrm{e}-14$ & 0.0000 & $4.8955681 \mathrm{e}-007$ & 0.0000 \\
\hline 2.6 & 0.0000 & $.1170229 \mathrm{e}-14$ & 0.0000 & $1.8198392 \mathrm{e}-007$ & 0.0000 \\
\hline 2.7 & 0.0000 & $.1847548 \mathrm{e}-15$ & 0.0000 & $6.6827968 \mathrm{e}-008$ & 0.0000 \\
\hline 2.8 & 0.0000 & $.2885836 \mathrm{e}-16$ & 0.0000 & $2.4263568 \mathrm{e}-008$ & 0.0000 \\
\hline . & . & . & . & & . \\
\hline 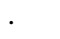 & & 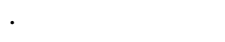 & . & & . \\
\hline 4.5 & 0.0000 & $.1825783 \mathrm{e}-30$ & 0.0000 & $2.4339438 \mathrm{e}-016$ & 0.0000 \\
\hline 4.6 & 0.0000 & $.2545821 \mathrm{e}-31$ & 0.0000 & $7.8413028 \mathrm{e}-017$ & 0.0000 \\
\hline 4.7 & 0.0000 & $.3536487 \mathrm{e}-32$ & 0.0000 & $2.5162744 \mathrm{e}-017$ & 0.0000 \\
\hline 4.8 & 0.0000 & $.4894037 \mathrm{e}-33$ & 0.0000 & $8.0416387 \mathrm{e}-018$ & 0.0000 \\
\hline 4.9 & 0.0000 & $.6657015 e-34$ & 0.0000 & $2.5139569 \mathrm{e}-018$ & 0.0000 \\
\hline 5 & 0.0000 & 0.0000 & 0.0000 & 0.0000 & 0.0000 \\
\hline
\end{tabular}




\subsection{Hyperbolic- parabolic- reaction problem:}

The second test problem is an unsteady hyperbolic- parabolicreaction problem described by (see Chen et al. (1998)).

$$
\frac{\partial \psi}{\partial t}+v \frac{\partial \psi}{\partial x}-D \frac{\partial^{2} \psi}{\partial x^{2}}=(3 v-9 D-1) \psi \quad, 0 \leq x \leq 1, v=0.3, D=0.002
$$

with initial condition

$$
\psi(x, 0)=e^{3 x} \quad, \quad 0 \leq x \leq 1,
$$

and boundary conditions

$$
\psi(0, t)=e^{-t} \quad \text { and } \quad \psi(1, t)=e^{3-t}
$$

The exact solution of this problem is $\psi(x, t)=e^{3 x-t}$

Figures $(7,8)$ show the numerical solutions of schemes (3.1) and (3.2) without transform of the problem (4.2). Also figures $(9,10)$ illustrate the numerical solutions with transform equation (4) which reveal that good results have been obtained. That is when we increase the value of time $t$ we get a smooth curve by using exponential transformation method which means that it is more accurate than without this transform. And we obtain that the Crank-Nicholson scheme is more accurate than Forward scheme. 


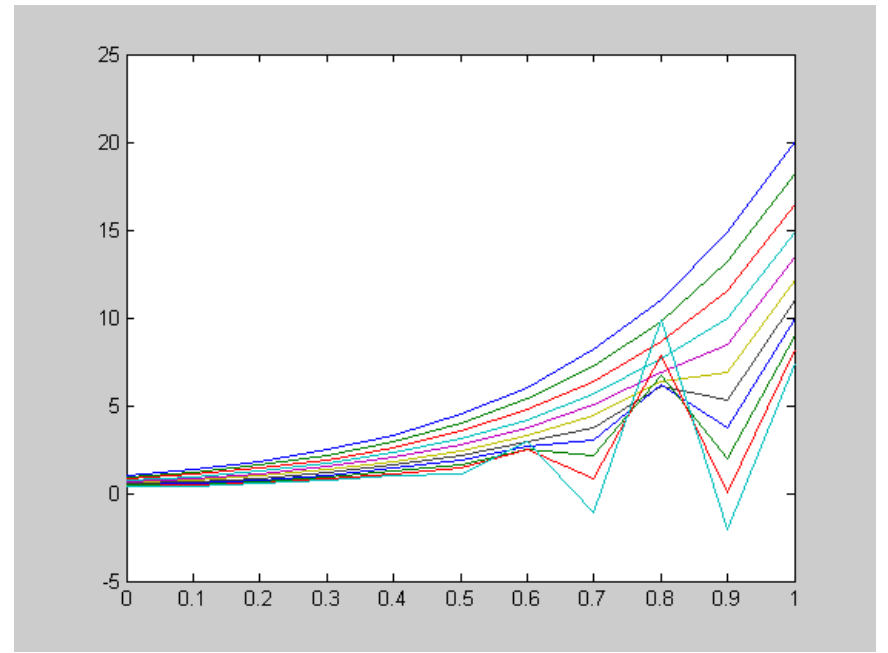

Fig.(7): Numerical approximation of the Forward F.D.M. for the second problem

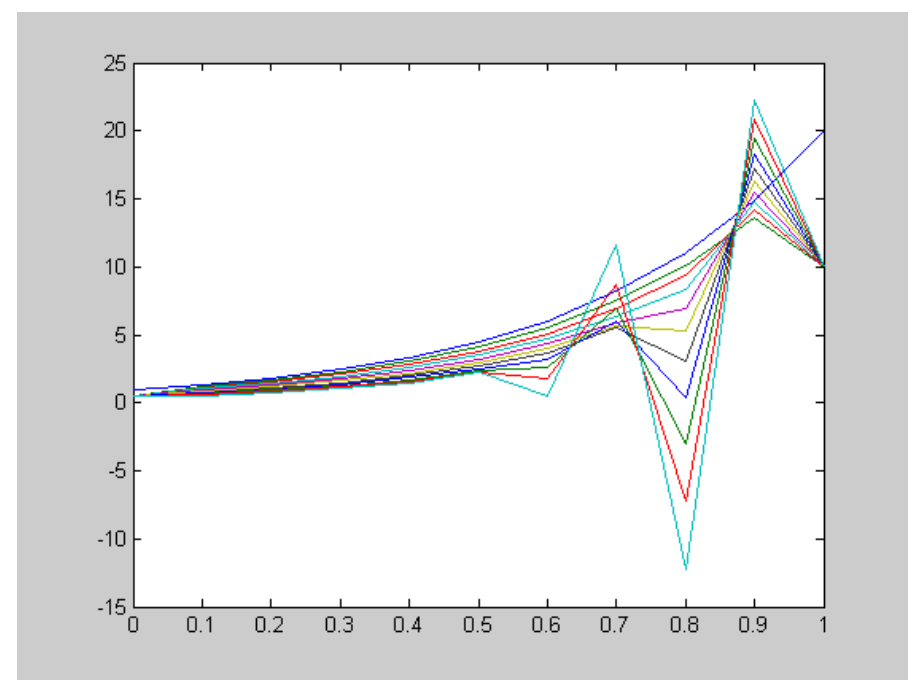

Fig.(8): Numerical approximation of the Crank-Nicolson F.D.M for the second problem 


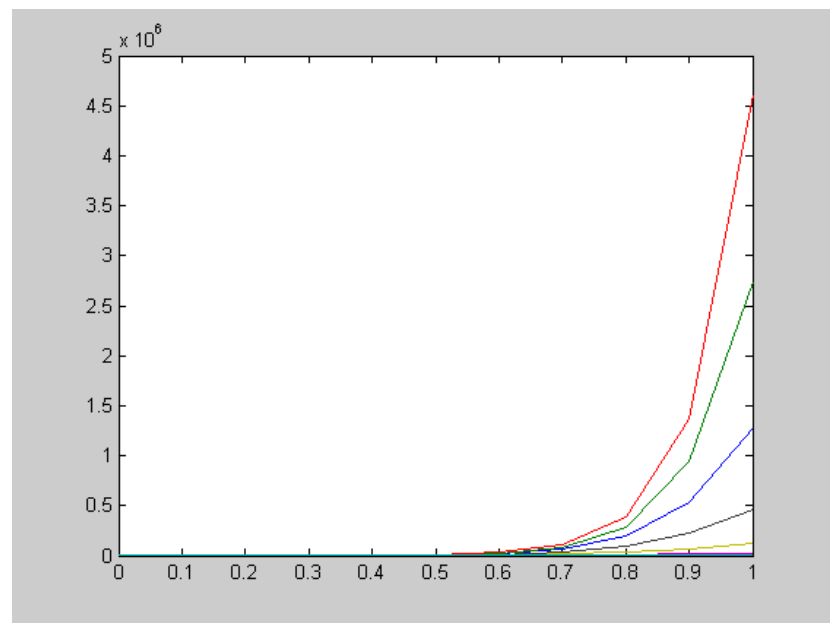

Fig.(9):Numerical approximation of the Forward F.D.M for the transformed second problem

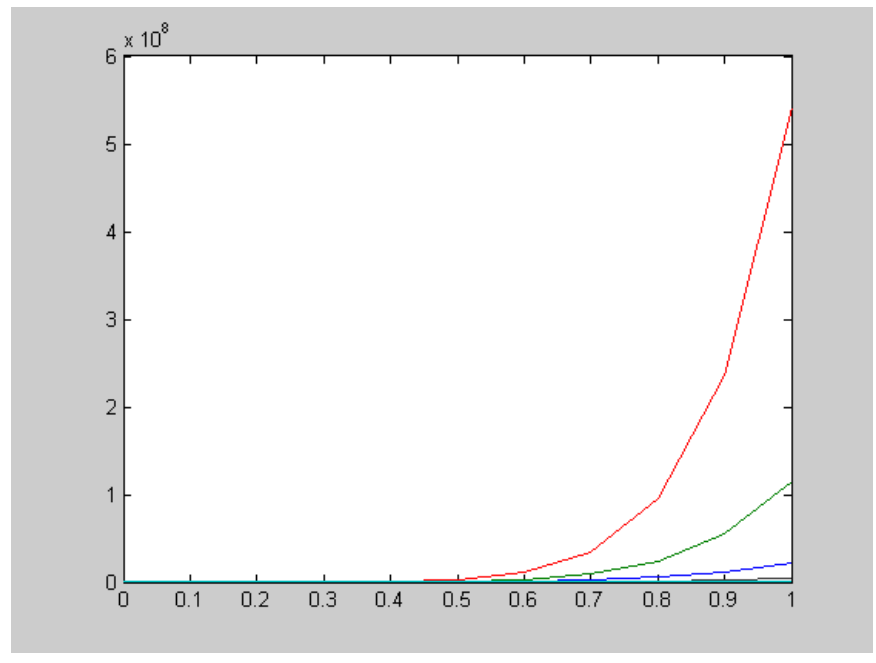

Fig.(10): Numerical approximation of the Crank-Nicolson F.D.M for the transformed second problem 


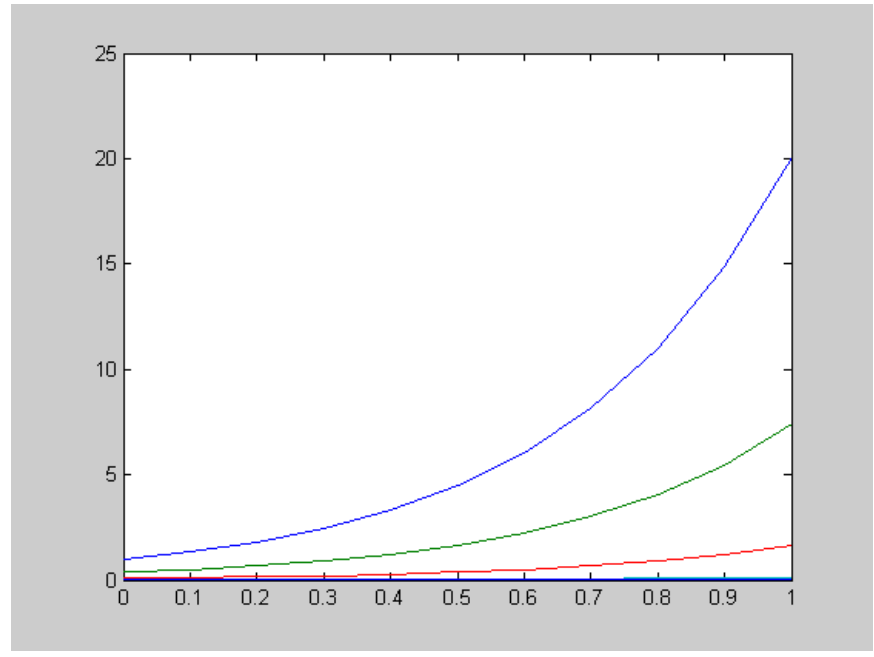

Fig. (11): The exact solution of the second problem

\section{Conclusions:}

The finite difference methods with the exponential transformation form are used to solve the classical differential representation for onedimensional hyperbolic- parabolic- reaction problems, and employed difference approximation techniques to obtain the numerical solutions.

Two different types of problems, hyperbolic-parabolic, and hyperbolic- parabolic- reaction, have been tested. We have compared between them using the exponential transformation form in eq.(4), we compared the results with the exact solutions which shows good numerical approximations in both examples. We have also obtained that the CrankNicholson scheme is more accurate than Forward scheme. Therefore the form of exponential transformation for the problem yields a stable solution compared with the exact solution. That is when we increase the value of time $\mathrm{t}$ we get a smooth curve by using exponential transformation method which means that it is more accurate than without this transform. 


\section{REFERENCES}

[1] Abramowitz, M. and Stegun, I. (1965) Handbook of Mathematical Functions. John Wiley and Sons Inc.

[2] Bindu, P.S. and Lakshmanan, M. (2002) "Symmetries and Integrability Properties of Generalized Fisher Type Nonlinear Diffusion Equation". Proceeding of Institute of Mathematics of NAS of Ukraine, vol. 43, part 1, pp. 36-48.

[3] Chen, B.M., Wo, S., and Wang, J. (1998) A High-Order Godunov Method for One Dimensional Convection-DiffusionReaction Problems, its supported in part by National science Foundation Grant.

[4] Garbey, M.; Kaper, H.G., and Romanyukha, N. (2001) "A Fast Solver for Systems of Reaction-Diffusion Equations", Thirteenth International Conference on Domain Decomposition Methods, Editors: Debit, N. ; Garbey ,M. , Hoppe , R. ; Periaus, J. ; Keses , D. ; and Knznetsov , pp.385-392.

[5] Guymon, G.L. (1970) "A Finite Element Solution of The OneDimensional Diffusion-Convection Equation". Water Resources Research, vol. 6, No. 1, pp. 204-210.

[6] Karamouzis, D.N. (1990) "A space Discontinuous Galerkin Method for one Dimensional Unsteady Convection-Diffusion Equation". Journal of Water Resources Management, vol.4, No. 4.

[7] Peaceman, D.W.(1977) Fundamentals of numerical reservoir simulation, New York: Elsevier Scientific Publishing Company.

[8] Riddaway, R.W. (2002) Numerical Methods. Meteorological Training Course Lecture Series, Revised by Hortal.

[9] Simth, I.M.; Farraday, R.V., and O,Connor, B.A. (1973) "RayleighRitz and Galerkin Finite Elements for Diffusion-Convection Problems". Water Resources Research, vol.9, No.3, pp. 593-606. 